\title{
ALIMENTAÇÃO COMPLEMENTAR DE LACTENTES ATENDIDOS EM UMA UNIDADE BÁSICA DE SAÚDE DA FAMÍLIA NO NORDESTE BRASILEIRO
}

\author{
Edcarla da Silva de Oliveira ${ }^{1}$, Veridianne Vasconcelos Ponte Viana ${ }^{2}$, Thábyta Silva Araújo ${ }^{3}$, Mariana \\ Cavalcante Martins ${ }^{4}$, Maria Vera Lúcia Moreira Leitão Cardoso ${ }^{4}$, Luisa Maria Oliveira Pinto ${ }^{6}$
}

\begin{abstract}
RESUMO: Objetivos: identificar a oferta de alimentos complementares às crianças com idade inferior a dois anos, e sua conformidade com o Ministério da Saúde. Método: estudo realizado com 52 crianças em uma Unidade Básica de Saúde do Nordeste brasileiro, entre dezembro/2015 e fevereiro/2016. Realizou-se entrevistas com as mães aplicando-se um questionário socioeconômico e relacionado ao perfil alimentar infantil. Analisou-se os dados no programa estatístico $\mathbf{R}^{\circledR}$. Classificou-se os alimentos consumidos em "não adequado" e "adequado" segundo o Ministério da Saúde. Resultados: dos maiores de 1 ano, uma (3,3\%) estava em aleitamento complementar, já os menores de 1 ano ( $n=22 / 100 \%)$ faziam uso de mingau e 20 (90,9\%) haviam consumido refrigerantes. Refrigerante, mingau, salgadinhos e biscoitos foram os alimentos mais consumidos. A alimentação "inadequada" prevaleceu nos menores de 1 ano. Conclusão: a alimentação é em sua maioria de produtos industrializados, tornando-se um problema de insegurança alimentar para a população do estudo.

DESCRITORES: Enfermagem; Alimentação complementar; Lactente; Educação em Saúde; Nutrição do Lactente.

\section{COMPLEMENTARY FOODS FOR INFANTS ATTENDED IN A FAMILY HEALTH CENTER IN THE NORTH EAST OF BRAZIL}

ABSTRACT: Objectives: To identify the offering of complementary foods to children aged below two years old, and the conformity of this with the Ministry of Health's recommendations. Method: a study undertaken with 52 children in a Primary Healthcare Center in the North East of Brazil, between December 2015 and February 2016. Interviews were held with the mothers, who were given a socioeconomic questionnaire which was also related to the children's dietary profile. The data were analyzed using the statistical program $\mathrm{R}^{\otimes}$. The foods consumed were classified as either "not appropriate" or "appropriate", according to the Ministry of Health. Results: of the children older than one year, one (3.3\%) was receiving complementary breastfeeding, while those younger than one year old $(n=22 / 100 \%)$ were eating baby porridge ('mingau' $)^{1}$ and $20(90.9 \%)$ had already drunk soda. Soda, mingau, salted snacks and cookies were the foods consumed most. "Inappropriate" food was prevalent in the children younger than one year old. Conclusion: most of the food given consisted of industrialized products, and is thus a problem of food insecurity for the study population.

DESCRIPTORS: Nursing; Complementary Feeding; Infant; Health Education; Infant Nutrition.
\end{abstract}

\section{ALIMENTACIÓN COMPLEMENTARIA DE LACTANTES ATENDIDOS EN UNA UNIDAD BÁSICA DE SALUD DE LA FAMILIA EN NORDESTE DE BRASIL}

RESUMEN: Objetivo: Identificar la oferta de alimentos complementarios a los niños con edad inferior a dos años, y su conformidad con el Ministerio de la Salud. Método: estudio realizado con 52 niños de una Unidad Básica de Salud de Nordeste brasileño, entre diciembre/2015 y febrero/2016. Se realizaron entrevistas con las madres por medio de cuestionario socioeconómico asociándolo al perfil alimentar infantil. Se analizaron los datos por medio del programa estadístico $R^{\circledR}$. Se clasificaron los alimentos consumidos en "no adecuado" y "adecuado" de acuerdo al Ministerio de la Salud. Resultados: De los niños con más de 1 año, un (3,3\%) estaba en amamantamiento complementario; ya los con menos de 1 año ( $n=22 / 100 \%)$ usaban papilla y 20 (90,9\%) habían consumido sodas. Soda, papilla, snacks y biscochos fueron los alimentos más consumidos. La alimentación "inadecuada" prevaleció para los menores de 1 año. Conclusión: Predomina la alimentación con productos industrializados, constituyendo un problema de inseguridad alimentar para la población del estudio.

DESCRIPTORES: Enfermería; Alimentación complementaria; Lactante; Educación en Salud; Nutrición del Lactante.

${ }^{1}$ Enfermeira. Mestranda em Enfermagem. Universidade Federal do Ceará. Canindé, CE, Brasil.

${ }^{2}$ Enfermeira. Mestranda em Enfermagem. Maternidade Escola Assis Chateaubriand da Universidade Federal do Ceará. Fortaleza, CE, Brasil.

${ }^{3}$ Enfermeira. Doutoranda em Enfermagem. Universidade Federal do Ceará. Fortaleza, CE, Brasil.

${ }^{4}$ Enfermeira. Doutora em Enfermagem. Docente de Enfermagem da Universidade Federal do Ceará. Fortaleza, CE, Brasil.

${ }^{5}$ Nutricionista. Mestre em Saúde Pública. Secretaria de Saúde do Estado do Ceará. Fortaleza, CE, Brasil. 


\section{INTRODUÇÃO}

A Organização Mundial de Saúde (OMS) e o Ministério da Saúde preconizam que a criança seja amamentada exclusivamente com leite materno até o sexto mês de vida ${ }^{(1-2)}$. A partir do sexto mês, há necessidade da introdução de alimentos que complementem o leite materno, pois nesse período somente o leite não é suficiente para suprir as necessidades da criança ${ }^{(2)}$.

A alimentação complementar é definida como o período em que outros alimentos ou líquidos são oferecidos em adição ao leite materno ${ }^{(2)}$. Qualquer alimento oferecido ao lactente além do leite materno, durante esse período, é chamado de alimento complementar ${ }^{(3-4)}$. Porém, observa-se que parte das mães recorre a outros métodos alimentares de fácil acesso e preparo durante o processo de adaptação alimentar do bebê $\hat{e}^{(5)}$.

Estudos mostram que a alimentação em nossa sociedade é de baixa qualidade, apresentando consumo frequente de leite de vaca, farinhas, achocolatados, açúcares, salgadinhos, refrigerantes e doces, o que repercute negativamente na primeira infância ${ }^{(6)}$. O Instituto Brasileiro de Geografia e Estatística (IBGE) constatou em 2015 que a obesidade infantil triplicou (9,7\% para 33,5\%) nos últimos 40 anos $^{(7)}$. Uma em cada três crianças sofre com a doença no Brasil e projeções da OMS apontam que, até 2025, o número de crianças com sobrepeso e obesidade pode chegar a 75 milhões, caso nada seja feito $^{(1)}$.

A obesidade acarreta complicações orgânico-funcionais, como doenças crônico-degenerativas (diabetes, hipertensão arterial, doenças coronarianas), e psicossociais, como afastamento das relações sociais, vergonha, exclusão e distúrbios na consciência corporal ${ }^{(8)}$. A obesidade é considerada um problema de saúde pública mundial; no caso das crianças, nos últimos 10 anos, houve um aumento de $40 \%$ nos índices de obesidade infantil( ${ }^{(9)}$. Em 2015 28,1\% das crianças entre 2 e 5 anos da região Nordeste apresentaram excesso de peso ${ }^{(7-9)}$.

Para classificação do estado nutricional das crianças, o Ministério da Saúde adota as curvas de crescimento e desenvolvimento infantil da OMS, as quais classificam as crianças entre 0 e 5 anos segundo a relação peso/idade, através de percentis: baixo peso (IMC $<$ P3), peso normal $(\mathrm{P} 3<\mathrm{IMC}<\mathrm{P} 85)$, sobrepeso $(85 \leq \mathrm{IMC}<\mathrm{P} 97)$ e obesidade ${ }^{(1)}$.

A obesidade, atualmente, é um dos grandes desafios da saúde pública, inclusive na pediatria, desde os lactentes até a adolescência. Nesse contexto, sabe-se que os primeiros meses de vida são apontados como cruciais para o desenvolvimento da obesidade ${ }^{(10)}$. Por esse motivo, a alimentação complementar saudável vem se tornando uma prioridade nas políticas públicas de alimentação e saúde no Brasil ${ }^{(11)}$.

Para a alimentação complementar ser aplicada de forma mais adequada, as mães necessitam de orientações acerca do assunto ${ }^{(5)}$. A desinformação e as orientações prestadas de forma insatisfatória podem comprometer significativamente o processo de introdução da alimentação complementar à criança e a manutenção do aleitamento materno até os dois anos de idade ou mais, conforme preconizado pela OMS e Ministério da Saúde ${ }^{(1-2)}$.

Os profissionais devem buscar estratégias para conhecer melhor as mudanças de comportamento provenientes das próprias mães, aprimorando e integrando ações de práticas alimentares saudáveis, principalmente nos serviços de atenção primária com vistas à prevenção de maiores agravos nutricionais, como sobrepeso ou obesidade, por meio de intervenções que priorizem e melhorem a qualidade da alimentação complementar da criança a partir do sexto mês de vida ${ }^{(5,11)}$.

Por esse motivo, surgiram os seguintes questionamentos: quais complementos alimentares estão sendo ofertados aos lactentes que estão em acompanhamento na Atenção Básica de Saúde num município do nordeste brasileiro? Torna-se relevante essa pesquisa, pois se observou que diversos estudos apontaram a fase de transição/complementação alimentar e em decorrência o aumento de peso na infância, como algo que merece grande atenção tanto por parte dos familiares, quanto dos profissionais de saúde que assistem a criança ${ }^{(4-5)}$.

A alimentação na infância compõe um dos aspectos essenciais para a manutenção da saúde infantil, sendo imprescindível que, nos dois primeiros anos de vida, sejam adotados hábitos alimentares 
saudáveis. O ato de se alimentar no começo da vida é delicado, e deve ser levado em consideração, pois serão esses hábitos alimentares que perdurarão pelo resto da vida ${ }^{(11)}$.

Diante do exposto, este estudo teve como objetivo identificar os alimentos complementares ofertados pelas mães que amamentam em uma Unidade Básica de Saúde da Família de acordo com o preconizado pelo Ministério da Saúde, bem como estabelecer a relação de risco para sobrepeso/ obesidade nessas crianças.

\section{- MÉTODO}

Estudo do tipo descritivo, transversal, quantitativo, realizado em uma Unidade Básica de Saúde da Família, em Canindé, município do interior do Ceará/Nordeste brasileiro e que possui uma população de 74.486 habitantes $^{(12)}$. Escolheu-se essa Unidade por ser a Unidade de Saúde da sede do município com maior número de famílias cadastradas (6.273) no ano de 2016.

Para o cálculo amostral, considerou-se os dados do Sistema de Informação da Atenção Básica (SIAB), sendo que o universo de crianças na faixa etária pretendida - de 6 meses a menores de 2 anos - perfez um total de 98 indivíduos. Estabeleceram-se os seguintes critérios de inclusão: crianças que estivessem em aleitamento complementar e mães que fossem responsáveis pelo preparo da alimentação da criança durante o dia. Os critérios de exclusão foram: mães que não tinham convívio direto com a criança e que não experienciaram a amamentação por ordem de saúde ou outros motivos que impedissem a aleitamento. Após critérios estabelecidos, a amostra constou de 52 binômios mãe-filho.

Os dados foram coletados entre os meses de dezembro de 2015 e fevereiro de 2016, por meio de entrevista semiestruturada com mães, aplicada pela pesquisadora do estudo na referida Unidade de Saúde, durante as consultas de puericultura. Utilizou-se um instrumento já validado e publicado em 2015(3), composto de 58 questões fechadas, que abrangiam dados sociodemográficos da mãe/cuidador principal e da criança, bem como perguntas relacionadas aos hábitos alimentares da criança e seu consumo alimentar em um período de 24 horas.

Das questões abordadas que faziam parte da entrevista às mães, na parte de inquérito alimentar da criança foram escolhidas um total de 11 questões, que versavam sobre os principais alimentos consumidos pelos menores de 2 anos no seu dia-a-dia. Os alimentos foram classificados em adequados e não adequados de acordo com o manual de referência do Ministério da Saúde, que trata do aleitamento materno e da alimentação infantil(2). Os dados foram classificados de acordo com a idade das crianças, separadas por faixa etária: menores de 1 ano de vida e maiores de 1 ano de vida, e os alimentos segundo o que cada um podia consumir em sua faixa etária. Os menores de 1 ano de idade eram 22 crianças e os maiores de 1 ano 30 crianças.

$\mathrm{Na}$ análise dos dados, elencaram-se os alimentos como sendo adequados: leite materno, frutas, verduras, legumes, alimentos ricos em proteínas, cálcio e derivados magros do leite; e não adequados: frituras, refrigerantes, alimentos ricos em gorduras, carboidratos, corantes e açúcares e alimentos ultraprocessados ${ }^{(2)}$.

Após a categorização dos alimentos utilizados, fez-se uma recategorização em duas variáveis: risco para sobrepeso/obesidade como presente ou ausente, sendo assim definidas: risco presente - quando as mães ofertaram seis ou mais alimentos classificados como não adequados na classificação citada anteriormente; risco ausente - os que tiveram menos de seis alimentos não adequados ofertados nas $24 \mathrm{~h}$ do recordatório, de acordo com a classificação anterior. Ressalta-se que foi utilizado como critério o fator presente maior que seis respostas, tomando como base a metade do número de questões mais uma, seguindo assim o pressuposto de variável bivariada.

Os dados foram compilados em um banco de dados com auxílio de uma planilha eletrônica, no programa Microsoft Excel 2007 e posteriormente alocadas e analisadas no programa $\mathrm{R}^{\circledast}$ versão 64.1. Analisaram-se as frequências absolutas e relativas, para observação do percentual de alimentos ofertados às crianças. Realizou-se teste de diferença de proporção de médias, para estabelecimento do risco de sobrepeso/obesidade. Para o cálculo da correlação entre as variáveis do estudo foi utilizado o teste qui-quadrado de Pearson. Foi adotado $\mathrm{p}<0,05$ como nível de significância. Foram calculados os 
intervalos com $95 \%$ de confiança.

As mães assinaram Termo de Consentimento Livre e Esclarecido (TCLE). O estudo foi aprovado pelo Comitê de Ética e Pesquisa da Escola de Saúde Pública do Ceará via Plataforma Brasil, com o número do parecer 1.350.061.

\section{- RESULTADOS}

Das crianças, 29 (53,8\%) são do sexo feminino. A média de idade foi de 11,9+5,02 meses, sendo a média de peso atual de 9,5+1,99 quilogramas. Ao analisar as curvas de peso, segundo os percentis da OMS, 25 crianças já se encontravam na faixa do sobrepeso/obesidade. A média de aleitamento materno exclusivo foi de 3,7 meses, sendo predominante em 19 dos menores de 1 ano de idade (83,4\%).

A média da idade materna foi de 26,8 7 7,32 anos, sendo que 27 tinham mais de 25 anos de idade e 30 haviam estudado 8 anos ou mais (57,7\%). Quanto à ocupação materna, 14 (26,9\%) mães referiram trabalhar fora de casa e 45 (86,5\%) coabitavam com companheiro. Das mães, 26 (48,8\%) eram primíparas e $43(82,7 \%)$ referiram ter amamentado a criança na primeira hora de vida.

As Tabelas 1 e 2 apresentam os percentuais dos alimentos consumidos por crianças na faixa etária menor de 1 ano e maior de 1 ano. Realizou-se teste de diferença de proporção de médias para observar o grau de significância entre os dados a seguir.

Tabela 1 - Consumo alimentar dos lactentes menores de 1 ano de idade, de acordo com preconizado pelo Ministério da Saúde. Canindé, CE, Brasil, 2016

\begin{tabular}{lccccc} 
Variáveis alimentares & \multicolumn{2}{c}{ Adequado } & \multicolumn{2}{c}{ Não adequado } \\
\cline { 2 - 6 } & $\mathbf{N}$ & $\mathbf{\%}$ & $\mathbf{n}$ & $\mathbf{\%}$ & $\mathbf{p}$ valor \\
\hline Leite de peito & 19 & 83,4 & 3 & 13,6 & $\mathbf{0 , 0 0 1}$ \\
\hline Chá & 15 & 68,2 & 7 & 31,8 & 0,135 \\
\hline Leite em pó e líquido de vaca & 3 & 13,4 & 19 & 83,4 & $\mathbf{0 , 0 0 1}$ \\
\hline Suco industrializado & 11 & 50 & 11 & 50 & 1 \\
\hline Refrigerante & 2 & 9,1 & 20 & 90,9 & $<\mathbf{0 , 0 0 1}$ \\
\hline Mingau & 0 & - & 22 & 100 & $<\mathbf{0 , 0 0 1}$ \\
\hline Açúcar e seus derivados & 10 & 45,4 & 13 & 59,1 & 0,831 \\
\hline Fruta in natura & 19 & 83,4 & 3 & 13,6 & 0,001 \\
\hline Doces e guloseimas (pirulitos/balas) & 4 & 18,2 & 18 & 81,8 & $\mathbf{0 , 0 0 5}$ \\
\hline Biscoitos recheados e salgadinhos & 7 & 31,8 & 15 & 68,2 & 0,135 \\
\hline Alimentos ultraprocessados (salsicha, linguiça, nuggets) & 13 & 59,1 & 9 & 40,9 & 0,522 \\
\hline Macarrão instantâneo & 10 & 45,5 & 12 & 54,5 & 0,831
\end{tabular}

Tabela 2 - Consumo alimentar dos lactentes maiores de 1 ano de idade, de acordo com preconizado pelo Ministério da Saúde. Canindé, CE, Brasil, 2016 (continua)

\begin{tabular}{lccccc} 
Variáveis alimentares & \multicolumn{2}{c}{ Adequado } & \multicolumn{2}{c}{ Não adequado } \\
\cline { 2 - 6 } & $\mathbf{N}$ & $\mathbf{\%}$ & $\mathbf{n}$ & $\mathbf{\%}$ & $\mathbf{p ~ v a l o r ~}$ \\
\hline Leite de peito & 1 & 3,3 & 29 & 96,7 & $<\mathbf{0 , 0 0 1}$ \\
\hline Chá & 14 & 46,7 & 16 & 53,3 & 0,855 \\
\hline Leite em pó e líquido de vaca & 29 & 96,7 & 1 & 3,3 & $<\mathbf{0 , 0 0 1}$ \\
\hline Suco industrializado & 16 & 53,3 & 14 & 46,7 & 0,855 \\
\hline Refrigerante & 14 & 46,7 & 16 & 53,3 & 0,201 \\
\hline Mingau & 19 & 63,3 & 11 & 36,7 & 0,001 \\
\hline \hline
\end{tabular}




\begin{tabular}{lccccc}
\hline Açúcar e seus derivados & 24 & 80 & 11 & 36,7 & 0,001 \\
\hline Fruta in natura & 4 & 13,3 & 26 & 86,7 & $<\mathbf{0 , 0 0 1}$ \\
\hline Doces e guloseimas (pirulitos/balas) & 20 & 66,7 & 10 & 33,3 & 0,1 \\
\hline Biscoitos recheados e salgadinhos & 29 & 96,7 & 1 & 3,3 & $<\mathbf{0 , 0 0 1}$ \\
\hline Alimentos ultraprocessados (salsicha, linguiça, nuggets) & 7 & 23,3 & 23 & 76,7 & 0,006 \\
\hline Macarrão instantâneo & 19 & 63,3 & 11 & 36,7 & 0,201
\end{tabular}

O teste apresentou significância prevalente para risco de sobrepeso/obesidade nos menores de 1 ano, quanto à alimentação não adequada. Mesmo o aleitamento materno sendo predominante em ambas as faixas etárias, a média de oferta de outros leites foi de 3,25 mamadeiras ao dia e a de mingau 2,71 mamadeiras. A fruta in natura foi um dos alimentos mais ofertados em ambas as faixas etárias, porém, seu consumo não chegou a duas porções ao dia (1,53 porções). Merece destaque também o uso de chás com fins medicinais, em 16 das 30 crianças maiores de 1 ano (46,7\%). Da amostra total, 28 crianças faziam uso de suco em pó (53,3\%) e 27 de caixinha (51,9\%), obtendo média nos menores de 1 ano.

Em relação ao risco para obesidade/sobrepeso correlacionando as variáveis maternas, as mães solteiras com crianças menores de 1 ano obtiveram análise estatística significativa para $\mathrm{risco}\left(\mathrm{p} / \mathrm{x}^{2}=0.012\right)$. Ao analisar-se apenas o percentual de risco presente, as variáveis idade, estado civil, escolaridade e número de filhos apresentaram risco homogêneo $(27,27 \%)$.

Nas demais não se observou significância estatística quando se avaliou o risco de desenvolver sobrepeso/obesidade entre as crianças menores de 1 ano, de acordo com o valor $p$ de Qui-quadrado.

Tabela 3 - Risco de obesidade/sobrepesos dos lactentes menores de 1 ano de idade segundo as variáveis sociodemográficas maternas. Canindé, CE, Brasil, 2016

\begin{tabular}{|c|c|c|c|c|c|c|}
\hline \multirow{2}{*}{$\begin{array}{l}\text { VARIÁVEIS MATERNAS } \\
\text { IDADE }\end{array}$} & \multirow{2}{*}{$\frac{\text { TOTAL }}{\mathrm{N}}$} & \multicolumn{2}{|c|}{ RISCO AUSENTE } & \multicolumn{2}{|c|}{ RISCO PRESENTE } & \multirow[t]{2}{*}{$x^{2}$} \\
\hline & & $\mathrm{N}$ & $\%$ & $\mathrm{n}$ & $\%$ & \\
\hline$<25$ anos & 10 & 7 & 31,8 & 3 & 13,6 & 1 \\
\hline$>25$ anos & 12 & 9 & 40,9 & 3 & 13,6 & \\
\hline \multicolumn{7}{|l|}{ ESTADO CIVIL } \\
\hline Solteira & 4 & 1 & 4,5 & 3 & 13,6 & 0,012 \\
\hline Casada & 18 & 15 & 68,2 & 3 & 13,6 & \\
\hline \multicolumn{7}{|l|}{ ESCOLARIDADE } \\
\hline Ensino fundamental & 5 & 4 & 18,2 & 1 & 4,5 & 0,057 \\
\hline Ensino médio & 17 & 12 & 54,5 & 5 & 22,7 & \\
\hline \multicolumn{7}{|l|}{ OCUPAÇÃO MATERNA } \\
\hline Trabalha fora de casa & 6 & 4 & 18,2 & 2 & 9,1 & 0,19 \\
\hline Não está trabalhando & 16 & 4 & 18,2 & 12 & 54,5 & \\
\hline \multicolumn{7}{|l|}{ NÚMERO DE FILHOS } \\
\hline Primeiro filho & 11 & 7 & 31,8 & 4 & 18,2 & 0,23 \\
\hline 2 ou mais filhos & 11 & 9 & 40,9 & 2 & 9,1 & \\
\hline
\end{tabular}

$\mathrm{x}^{2}=$ qui-quadrado $($ valor $\mathrm{p})$

As variáveis maternas das crianças maiores de 1 ano não apresentaram nenhuma associação estatisticamente significativa para o risco de desenvolver sobrepeso/obesidade. Ao analisar-se apenas a média simples da amostra, o maior percentual de risco presente esteve nas variáveis idade e ocupação materna $(50 \%)$, seguidas do número de filhos $(46,67 \%)$. 
Tabela 4 - Risco de sobrepeso/obesidade dos lactentes maiores de 1 ano de idade segundo as variáveis sociodemográficas maternas. Canindé, CE, Brasil, 2016

\begin{tabular}{|c|c|c|c|c|c|c|}
\hline \multirow{2}{*}{$\begin{array}{l}\text { VARIÁVEIS MATERNAS } \\
\text { IDADE }\end{array}$} & \multirow{2}{*}{$\frac{\text { TOTAL }}{\mathrm{N}}$} & \multicolumn{2}{|c|}{ RISCO AUSENTE } & \multicolumn{2}{|c|}{ RISCO PRESENTE } & \multirow[t]{2}{*}{$\mathbf{x}^{2}$} \\
\hline & & $\mathrm{n}$ & $\%$ & $\mathrm{n}$ & $\%$ & \\
\hline$<25$ anos & 15 & 8 & 26,7 & 7 & 23,4 & 1 \\
\hline$>25$ anos & 15 & 7 & 23,4 & 8 & 26,7 & \\
\hline \multicolumn{7}{|l|}{ ESTADO CIVIL } \\
\hline Solteira & 3 & 1 & 3,3 & 2 & 6,7 & \multirow[t]{2}{*}{0,138} \\
\hline Casada & 27 & 17 & 56,7 & 10 & 33,3 & \\
\hline \multicolumn{7}{|l|}{ ESCOLARIDADE } \\
\hline Ensino fundamental $(<8 a)$ & 17 & 12 & 40 & 5 & 16,7 & \multirow[t]{2}{*}{0,601} \\
\hline Ensino médio (>8a) & 13 & 8 & 26,7 & 5 & 16,7 & \\
\hline \multicolumn{7}{|l|}{ OCUPAÇÃO MATERNA } \\
\hline Trabalha fora de casa & 7 & 5 & 16,7 & 2 & 6,7 & \multirow[t]{2}{*}{0,387} \\
\hline Não está trabalhando & 23 & 10 & 33,3 & 13 & 43,3 & \\
\hline \multicolumn{7}{|l|}{ NÚMERO DE FILHOS } \\
\hline Primeiro filho & 14 & 7 & 23,4 & 7 & 23,34 & 0,613 \\
\hline 2 ou mais filhos & 16 & 10 & 33,3 & 6 & 20 & \\
\hline
\end{tabular}

$\mathrm{x}^{2}=$ qui-quadrado (valor $\left.\mathrm{p}\right)$

Fonte: Pesquisa do autor

\section{- DISCUSSÃO}

A manutenção do aleitamento materno ocorreu de forma mais prevalente nas crianças menores de 1 ano de vida. Dados semelhantes foram encontrados em diversas pesquisas onde a prevalência de aleitamento materno foi maior nas crianças menores de 12 meses: 46,7\%, 77,27\% e 59\% ${ }^{(5,12)}$. Estudos mais recentes apresentaram percentuais para aleitamento em maiores de 1 ano de $65,5 \%$ e $58,1 \%{ }^{(14-15)}$.

Ambas as faixas etárias apresentam índices menores se comparados com a média da população brasileira, que é de 7,6 meses para o aleitamento materno ${ }^{(15)}$. O abandono do aleitamento predispõe ao aparecimento de obesidade e doenças crônicas como o diabetes tipo I e a hipertensão arterial, além de morbidades como doenças diarreicas, infecciosas e do trato respiratório ${ }^{(13,16-17)}$.

O percentual de consumo de outros leites (em pó e líquido de vaca) foi prevalente nos $>$ de 1 ano e o uso de mingau foi de 78,8\% na amostra total. Das crianças menores de 1 ano, todas ingeriam mingau (100\%). Ambos apresentaram significância estatística $(p<0,001)$. Estudo realizado em cinco países europeus, com 746 crianças entre 6 e 24 meses, observou que os componentes lácteos são os mais comuns na dieta, sendo o principal contribuinte na oferta de proteínas ${ }^{(18)}$.

A introdução do leite integral de vaca e dos mingaus antes de 1 ano de vida são contraindicados, por serem responsáveis por $20 \%$ das alergias alimentares, o que pode desencadear doenças atópicas, como asma, micro-hemorragias intestinal, sobrecarga do sistema imune e renal, e desmame precoce. Além disso, apresentam baixos níveis de vitaminas $\mathrm{C}, \mathrm{D}$ e $\mathrm{E}$, quantidade inadequada de carboidratos e adição de outros açúcares. A partir dos 12 meses, a introdução desses alimentos deve ser vista com cautela $^{(13-14)}$.

O uso de refrigerantes foi significativo como fator de risco para desenvolvimento do sobrepeso/ obesidade nas crianças maiores de 1 ano $(p<0,001)$. No estado do Rio de Janeiro, 38\% das crianças menores de 1 ano bebiam refrigerantes e o consumo inicial ocorreu antes dos 3 meses em 66,7\%, em pesquisa realizada ${ }^{(19)}$. Outras pesquisas apontam o consumo significativo de refrigerantes em crianças menores de 1 ano $(55,4 \%, 69,5 \%, 37,6 \% \text { e 53,8\% })^{(13,18-20)}$. Esses dados convergem com os dados da pesquisa realizada. 
O consumo de refrigerantes pode levar a uma deficiência na absorção de ferro não heme e afetar adversamente a relação cálcio/fósforo. A maioria dos pais desconhece a real densidade energética e o teor de açúcar dessas bebidas ${ }^{(20)}$. É necessário alertá-los na consulta de puericultura que esses alimentos devem ser excluídos da alimentação das crianças devido à quantidade elevada de açúcares, corantes e substâncias proibidas pela OMS, apresentando potencial alergênico e contribuindo para o excesso de peso e aparecimento de cáries ${ }^{(13)}$.

O uso de açúcar e seus derivados esteve presente na maioria da amostra dos lactentes, sendo o consumo mais evidente nos maiores de 1 ano (80\%). Dados semelhantes foram encontrados em outras pesquisas $^{(21-22)}$.

A pesquisa evidenciou um consumo de biscoitos recheados e salgadinhos elevado, atingindo o percentual de $84,6 \%$ da amostra total estudada, sendo mais prevalente nos maiores de 1 ano $(96,7 \%)$. Esses alimentos podem irritar a mucosa gástrica, induzir alergias e levar a obesidade(4-6). Em outras pesquisas os percentuais foram $64 \%$ e $71,7 \%$ de consumo entre os menores de 2 anos, convergindo com nossa pesquisa. O consumo desses alimentos é mais comum em famílias de menor poder aquisitivo $^{(20-21)}$.

Os biscoitos recheados podem desencadear hiperatividade, irritabilidade e alergias. Alimentos industrializados não são recomendados pela OMS aos menores de 2 anos de idade. Estudos sugerem que a partir dos 12 meses as crianças estão mais suscetíveis às práticas alimentares não saudáveis e como consequência a obesidade, por conta da mídia exacerbada para o consumo dos industrializados e de uma "falsa praticidade"(19).

O consumo de alimentos ultraprocessados (embutidos e macarrão instantâneo) é fator preocupante nessa faixa etária. Estudo realizado em creches públicas de São Paulo mostra que 58,2\% das crianças nessa faixa etária consumiam esses alimentos no almoço ofertado na instituição ${ }^{(21)}$. Estudo realizado em Minas Gerais demonstra a associação do risco para doenças cardiovasculares (DCV) em crianças que fazem uso constante desses alimentos. Além das DCVs, as dislipidemias, hipertensão arterial e hiperglicemia têm sido observadas ${ }^{(22)}$. Os mesmos possuem alto teor de sódio e de lipídeos, e em nada acrescentam a alimentação infantil, trazendo como consequências o aumento de peso e anemia ferropriva ${ }^{(8,20-21)}$.

Por fim, ao avaliarmos as variáveis maternas e os fatores de risco para sobrepeso/obesidade segundo a alimentação das crianças, encontramos risco significativo para aqueles filhos de mães solteiras, com menos tempo de estudo e menores de 25 anos. Em outros estudos nacionais e internacionais, as práticas alimentares inadequadas na infância estiveram presentes nas mães com baixa escolaridade e idade materna inferior a 25 anos $^{(21-23)}$.

Mães com menos de 8 anos de estudo tendem a oferecer com maior frequência alimentos com maior teor calórico. Filhos de mãe com baixa escolaridade tendem a apresentar curvas de peso estagnadas ou ascendentes ${ }^{(5,24)}$.

A inserção da mulher no mundo do trabalho e o papel como chefe de família convergem para padrões dietéticos inadequados, com impacto na introdução de alimentos industrializados e ultraprocessados precocemente na dieta infantil(23). Estudo realizado em Florianópolis mostra que a atividade fora do lar é fator de risco para sobrepeso/obesidade ${ }^{(25)}$. Estudo australiano também demonstrou que filhos de mães que trabalham foram do lar tendem a desenvolver obesidade precocemente, por conta do consumo de junk food ${ }^{(22)}$.

A introdução desses alimentos na dieta das crianças é fator decisivo para predisposição à obesidade e desenvolvimento de doenças crônicas não transmissíveis na vida adulta ${ }^{(26)}$. Os dados apresentados descrevem uma alta prevalência de consumo precoce de alimentos industrializados. Ressalta-se a necessidade de desestimular o consumo desses alimentos e reforçar o de frutas e hortaliças ${ }^{(27)}$.

Tem-se como limitação do estudo o fato da amostra ter um número reduzido de participantes e o viés recordatório da mãe, já que muitas vezes ela pode omitir algum fato ou esquecê-lo.

\section{- CONCLUSÃo}


A prevalência de consumo alimentar não adequado foi mais visível na população menor de 1 ano. Os alimentos não adequados mais consumidos pela população foram o leite em pó e líquido de vaca, mingaus, refrigerantes, biscoitos recheados e salgadinhos. Essa alimentação, aliada à baixa escolaridade materna das mães e àquelas que trabalham fora de casa, mostraram-se como fatores para o risco de desenvolvimento do sobrepeso/obesidade.

Relacionado às práticas adequadas de alimentação, tem-se a prevalência e manutenção do aleitamento materno de forma complementar e o consumo da fruta in natura. A alimentação do grupo estudado é em sua maioria de produtos industrializados, tornando-se um problema de insegurança alimentar para a população do estudo, relacionado à Segurança e Alimentação Nutricional preconizada pelo Ministério da Saúde e OMS.

Com isso, remete-se à importância da ação dos profissionais de saúde, particularmente do enfermeiro(a), visto que tem em seu arcabouço de conhecimentos, fundamentos teóricos e práticos para a promoção da saúde no que concerne à alimentação complementar da criança e do acompanhamento da saúde infantil, envolvendo estratégias de educação e saúde, avaliação do crescimento e desenvolvimento e orientações considerando a realidade das famílias no contexto cultural e socioeconômico.

\section{REFERÊNCIAS}

1. World Health Organization (WHO). Multicentre Growth Reference Study Group. Child Growth Standards: Length/height-for-age, weight-for-age, weight-for-length, weight-for-height and body mass index-for-age: Methods and development. [Internet] WHO: Geneva; 2006 [acesso em 16 set 2015]. Disponível: http://www.who. int/childgrowth/standards/Technical_report.pdf?ua=1.

2. Ministério da Saúde (BR). Saúde da criança: Aleitamento materno e alimentação complementar. [Internet] Brasília:Ministério da Saúde; 2015 [acesso em 12 ago 2015]. Disponível: http://bvsms.saude.gov.br/bvs/publicacoes/ saude_crianca_aleitamento_materno_cab23.pdf.

3. Oliveira JM, de Castro IRR, Bioni e Silva G, Venancio SI, Saldiva SRDM. Avaliação da alimentação complementar nos dois primeiros anos de vida: proposta de indicadores e de instrumento. Cad. Saúde Pública. [Internet] 2015;31(2) [acesso em 22 mar 2016]. Disponível: http://dx.doi.org/10.1590/0102-311X00209513.

4. Bowden VR, Greenberg CS. Procedimentos de enfermagem pediátrica. $3^{\mathrm{a}}$ ed. Rio de Janeiro: Guanabara Koogan; 2013.

5. Carneiro GCS, de Morais LMC, Costa LFA, de Moura THM, Javorski M, Leal LP. Crescimento de lactentes atendidos na consulta de enfermagem em puericultura. Rev. Gaúcha Enferm. [Internet] 2015;36(1) [acesso em 23 out 2015]. Disponível: http://dx.doi.org/10.1590/1983-1447.2015.01.45703.

6. Ramires EKNM, de Menezes RCE, Oliveira JS, Oliveira MAA, Temoteo TL, Longo-Silva G, et al. Estado nutricional de crianças e adolescentes de um município do semiárido do Nordeste brasileiro. Rev. paul. pediatr. [Internet] 2014;32(3) [acesso em 20 dez 2015]. Disponível: http://dx.doi.org/10.1590/0103-0582201432309.

7. Instituto Brasileiro de Geografia e Estatística (IBGE). Pesquisa Nacional de Saúde 2013 - Percepção do estado de saúde, estilos de vida e doenças crônicas. [Internet] Rio de Janeiro: IBGE; 2014 [acesso em 30 mar 2016]. Disponível: ftp://ftp.ibge.gov.br/PNS/2013/pns2013.pdf.

8. Pimpin L, Jebb S, Johnson L, Wardle J, Ambrosini GL. Dietary protein intake is associated with body mass index and weight up to 5 y of age in a prospective cohort of twins. Am J Clin Nutr. [Internet] 2016;103(2) [acesso em 20 jan 2016]. Disponível: http://dx.doi.org/10.3945/ajcn.115.118612.

9. Associação Brasileira para Estudo da Obesidade e da Síndrome Metabólica (ABESO). Mapa da Obesidade no Brasil. [Internet] 2015 [acesso em 26 jan 2016]. Disponível: http://www.abeso.org.br/atitude-saudavel/mapaobesidade.

10. Nascimento VG, da Silva JPC, Ferreira PC, Bertoli CJ, Leone C. Aleitamento materno, introdução precoce de leite não materno e excesso de peso na idade escolar. Rev. paul. pediatr. [Internet] 2016;34(4) [acesso em 24 jun 2016] Disponível: http://dx.doi.org/10.1016/j.rppede.2016.05.002. 
11. Vítolo MR, Louzada ML, Rauber F, Grechi P, Gama CM. Impacto da atualização de profissionais de saúde sobre as práticas de amamentação e alimentação complementar. Cad. Saúde Pública. [Internet] 2014;30(8) [acesso em 20 mar 2016]. Disponível: http://dx.doi.org/10.1590/0102-311X00186913.

12. Instituto Brasileiro de Geografia e Estatística (IBGE). Censo IBGE. [Internet] 2010 [acesso em 10 mar 2016$].$ Disponível: http://mapasinterativos.ibge.gov.br/grade/default.html.

13. Teixeira GA, Ferreira TLS, Carvalho JBL, Andrade FB. Práticas alimentares dos lactentes. Saúde (Santa Maria). [Internet] 2015;41(2) [acesso em 01 mai 2016]. Disponível: http://dx.doi.org/10.5902/2236583415239.

14. de Freitas LG, Escobar RS, Cortés MAP, Faustino-Silva DD. Consumo alimentar de crianças com um ano de vida num serviço de atenção primária em saúde. Rev. Port. Saúde Pública. [Internet] 2016;34(1) [acesso em 01 mai 2016]. Disponível: https://doi.org/10.1016/j.rpsp.2015.10.001.

15. Ministério da Saúde (BR). Secretaria de Atenção à Saúde. Departamento de Ações Programáticas e Estratégicas. II Pesquisa de Prevalência de Aleitamento Materno nas Capitais Brasileiras e Distrito. [Internet] Brasília: Ministério da Saúde; 2009 [acesso em 20 mar 2016]. Disponível: http://bvsms.saude.gov.br/bvs/publicacoes/pesquisa_ prevalencia_aleitamento_materno.pdf.

16. Russell CG, Taki S, Azadi L, Campbell KJ, Laws R, Elliott R, et al. A qualitative study of the infant feeding beliefs and behaviours of mothers with low educational attainment. BMC Pediatr. [Internet] 2016;16(1) [acesso em 28 jun 2016]. Disponível: http://dx.doi.org/10.1186/s12887-016-0601-2.

17. Prentice P, Ong KK, Schoemaker MH, van Tol EA, Vervoort J, Hughes IA, et al. Breast milk nutrient content and infancy growth. Acta Paediatr. [Internet] 2016;105(6) [acesso em 28 jul 2016] Disponível: http://dx.doi.org/10.1111/ apa.13362.

18. Ferreira FS. Consumo de alimentos impróprios por crianças menores de dois anos e suas possíveis consequências. Revista da Universidade Vale do Rio Verde. [Internet] 2015;13(1) [acesso em 29 jan 2016]. Disponível: http://periodicos.unincor.br/index.php/revistaunincor/article/view/1843.

19. Sotero AM, Cabral PC, da Silva GAP. Fatores socioeconômicos, culturais e demográficos maternos associados ao padrão alimentar de lactentes. Rev. paul. pediatr. [Internet] 2015;33(4) [acesso em 22 mar 2016. Disponível: https://doi.org/10.1016/j.rpped.2015.03.006.

20. Frota MA, Casimiro CF, Bastos PO, Sousa Filho OA, Martins MC, Gondim APS. Conhecimento de mães acerca do aleitamento materno e complementação alimentar: pesquisa exploratória. Online Braz J Nurs. [Internet] 2013;12(1) [acesso em 29 ago 2015]. Disponível: http://www.objnursing.uff.br/index.php/nursing/article/ view/3890/html.

21. Toloni MHA, Longo-Silva G, Goulart RMM, Taddei JAAC. A. Introdução de alimentos industrializados e de alimentos de uso tradicional na dieta de crianças de creches públicas no município de São Paulo. Rev Nutr. [Internet] 2011;24(1) [acesso em 01 abr 2016]. Disponível: http://dx.doi.org/10.1590/S1415-52732011000100006.

22. Damianidi L, Gruszfeld D, Verduci E, Vecchi F, Xhonneux A, Langhendries JP, et al. Protein intakes and their nutritional sources during the first 2 years of life: secondary data evaluation from the European Childhood Obesity Project. Eur J Clin Nutr. [Internet] 2016;70(11) [acesso em 01 abr 2016]. Disponível: http://dx.doi.org/10.1038/ ejcn.2016.108.

23. Levy-Costa RB, Sichieri R, Pontes NS, Monteiro CA. Disponibilidade domiciliar de alimentos no Brasil: distribuição e evolução (1974-2003). Rev. Saúde Pública. [Internet] 2005;39(4) [acesso em 01 abr 2016]. Disponível: http://dx.doi.org/10.1590/S0034-89102005000400003.

24. Zong XN, Li H, Zhang YQ. Family-related risk factors of obesity among preschool children: results from a series of national epidemiological surveys in China. BMC Public Health. [Internet] 2015;(15) [acesso em 20 jan 2016]. Disponível: http://dx.doi.org/10.1186/s12889-015-2265-5.

25. Marzano JBT. Fatores condicionantes para introdução precoce de alimentos industrializados em crianças de 4 meses a 5 anos de vida [monografia]. Brasilia (DF): Universidade de Brasília; 2013.

26. Brasil LMBF, das Neves OMD, de Amorim CSC, Lopez FA, Arias EHL, Piani PPF. Introdução de alimentos complementares em lactentes. Rev. Para. Med. [Internet] 2012;26(1) [acesso em 20 mar 2016]. Disponível: http:// files.bvs.br/upload/S/0101-5907/2012/v26n1/a3082.pdf. 
27. Caetano MC, Ortiz TT, da Silva SGL, de Souza FIS, Sarni ROS. Alimentação complementar: práticas inadequadas em lactentes. J. Pediatr. (Rio J.). [Internet] 2010;86(3) [acesso em 20 mar 2016]. Disponível: http:// dx.doi.org/10.1590/S0021-75572010000300006. 